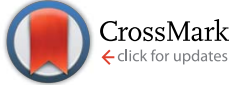

Cite this: RSC Adv., 2017, 7, 11135

Received 17th November 2016

Accepted 2nd February 2017

DOI: 10.1039/c6ra26958c

rsc.li/rsc-advances

\section{Multiscale modelling of heat conduction in all-MoS 2 single-layer heterostructures}

\author{
Bohayra Mortazavi ${ }^{\star a}$ and Timon Rabczuk ${ }^{b}$
}

Successful isolation of atom thick molybdenum disulfide $\left(\mathrm{MoS}_{2}\right)$ films has opened promising routes toward its practical applications in nanoelectronics. Recently, experimental fabrication of single-layer $\mathrm{MoS}_{2}$ membranes made from semiconducting $(2 \mathrm{H})$ and metallic (1T) phases was successfully accomplished in order to reach advanced $\mathrm{MoS}_{2}$ heterostructures with tunable electronic properties. A comprehensive understanding of the heat conduction properties of these heterostructures plays a crucial role not only for the overheating concerns in nanoelectronics but also for the design of specific systems such as thermoelectric nanodevices. In this investigation, we accordingly explore the thermal conductivity along all-MoS 2 heterostructures by developing a combined atomistic-continuum multiscale model. In this approach, molecular dynamics simulations were employed to compute the thermal conductivity of pristine $2 \mathrm{H}$ and $1 \mathrm{~T}$ phases and also the thermal contact conductance between $1 \mathrm{~T}$ and $2 \mathrm{H}$ phases. Properties obtained from the atomistic simulations were finally used to construct macroscopic samples of $\mathrm{MoS}_{2}$ heterostructures using the finite element method. Our investigation confirms the possibility of finely tuning the heat transport along $\mathrm{MoS}_{2}$ heterostructures by controlling the domain size and the concentration of different phases. Findings from our multiscale model provide useful insight regarding the thermal conduction response of all- $\mathrm{MoS}_{2}$ heterostructures.

\section{Introduction}

Graphene ${ }^{1-4}$ presents an exceptional combination of outstanding thermal conductivity, ${ }^{5}$ mechanical strength ${ }^{6}$ and electrical properties $^{4}$ which have promoted ongoing attention toward twodimensional (2D) materials. ${ }^{1-4,7-10}$ Nevertheless, the application of graphene in nanoelectronics is limited due to its zero-bandgap semiconducting properties. Hexagonal boron nitride (h-BN) $)^{11-13}$ is another high-quality $2 \mathrm{D}$ crystal experimentally available in the free-standing form, which is an electrical insulator with a large band-gap of $5.9 \mathrm{eV} .{ }^{11} \mathrm{We}$ note that graphene and h-BN both have honeycomb crystal structures with only $2 \%$ difference in their lattice constants. Thus, the fabrication of inplane seamlessly stitched heterostructures consisting of semimetal graphene and insulating h-BN, has already been proven to be an achievable route for tuning the electronic properties of graphene. ${ }^{\mathbf{1 4 , 1 5}}$ Since the fabrication of graphene and h-BN inplane heterostructures are complicated in practice, a more promising approach is to use materials with inherent semiconducting properties. In order to find a $2 \mathrm{D}$ material with semiconducting properties suitable for the nanoelectronics, most recently, transition metal dichalcogenides have attracted

${ }^{a}$ Institute of Structural Mechanics, Bauhaus-Universität Weimar, Marienstr. 15, D-99423 Weimar, Germany. E-mail: bohayra.mortazavi@gmail.com; Fax: +49 364 358 4511; Tel: +49 15780378770

${ }^{b}$ College of Civil Engineering, Department of Geotechnical Engineering, Tongji University, Shanghai, China.E-mail: Timon.rabczuk@uni-weimar.de considerable interest because of their unique electronic and magnetic properties compared to graphene and h-BN. Among the all members of the family of $2 \mathrm{D}$ transition metal dichalcogenides, molybdenum disulfide $\left(\mathrm{MoS}_{2}\right)^{16,17}$ has been so far the most attractive nanomembranes, not only because of its semiconducting properties with a finite and direct band-gap of $1.8 \mathrm{eV}$, but also due to the successful synthesis of large-area, high-quality and free-standing nanomembranes. Another appealing feature of $\mathrm{MoS}_{2}$ films is its polymorphic nature in which the atomic arrangement of $\mathrm{S}$ atoms can lead to contrasting electronic characteristics of semiconducting $(2 \mathrm{H})$ or metallic (1T) ${ }^{18-20}$ Amazingly, fabrication of $\mathrm{MoS}_{2}$ heterostructures made from metallic and semiconducting phases in the single-layer form has been accomplished successfully, ${ }^{18}$ which opens a practical prospect for further tuning the properties of $\mathrm{MoS}_{2}$.

One of the important properties for the design of nanodevices using the $2 \mathrm{D}$ materials, is the thermal conduction properties of the constituents which indicates how fast and efficient they can transmit heat. In particular, for many applications such as those related to the nanoelectronics and energy storage systems, overheating concerns demand for materials with high thermal conductivities in order to improve the thermal management of the systems. On the other side, for some applications like thermoelectrics, the suppressed thermal conductivity is more desirable to improve thermoelectric figure of merit and accordingly enhance the energy production efficiency. There exist numerous studies available in the literature 
with respect to the $\mathrm{MoS}_{2}$ heterostructures. ${ }^{21-24}$ Gao et al. ${ }^{21}$ reported that the phonon thermal transport in the graphene$\mathrm{MoS}_{2}$ bilayer heterostructure is reduced by the lattice mismatch. However, to the best of our knowledge, thermal conductivity of all-MoS $\mathrm{M}_{2}$ single-layer heterostructures have been studied neither theoretically nor experimentally. In the present investigation, we therefore intend to provide a general understanding concerning the heat conduction properties of all- $\mathrm{MoS}_{2}$ heterostructures by developing a combined atomistic-continuum multiscale modeling.

\section{Multiscale modeling}

The position of sulfur atoms in the atomic lattice of $\mathrm{MoS}_{2}$ nanomembranes not only substantially change its electronic properties ${ }^{18-20}$ but can also considerably affect its mechanical properties. $^{25}$ The most stable form of single-layer $\mathrm{MoS}_{2}$ is its semiconducting phase so called $2 \mathrm{H}$ which presents a hexagonal lattice with atomic stacking sequence of "ABA". On the other side, the $1 \mathrm{~T}$ phase which presents metallic characteristics present an atomic stacking sequence of "ABC", in which the $\mathrm{S}$ atoms on the top are placed in the hollow center of the hexagonal lattice. ${ }^{25}$ In Fig. 1a, schematic illustrations of $2 \mathrm{H}$ and $1 \mathrm{~T}$ are shown. An atomistic model of single layer $2 \mathrm{H} / 1 \mathrm{~T}$ heterostructure is also illustrated in Fig. 1b.

According to the experimental observations, ${ }^{18} 1 \mathrm{~T}$ or $2 \mathrm{H}$ phases inside the heterostructures present triangular geometries ${ }^{\mathbf{1 8}}$ with a characteristic domain size, which is represented in our study by " $D$ ". In the present investigation, we explored the thermal conductivity of different single-layer $\mathrm{MoS}_{2}$ heterostructures with various combination of $1 \mathrm{~T}$ and $2 \mathrm{H}$ phases and with different characteristic domain sizes as well. Based on the experimental observations, ${ }^{18}$ between $2 \mathrm{H}$ and $1 \mathrm{~T}$ phases depending on the configuration of heterostructure, 3 different grain boundaries namely; " $\alpha$ ", " $\beta$ " and " $\gamma$ " can be formed. The top and side views of atomic structure of these grain boundaries are shown in Fig. 2. In this work, we developed a program to create the atomistic models of these grain boundaries. Depending on the configuration of single-layer $\mathrm{MoS}_{2}$ heterostructure, the arrangement of different grain boundaries in the

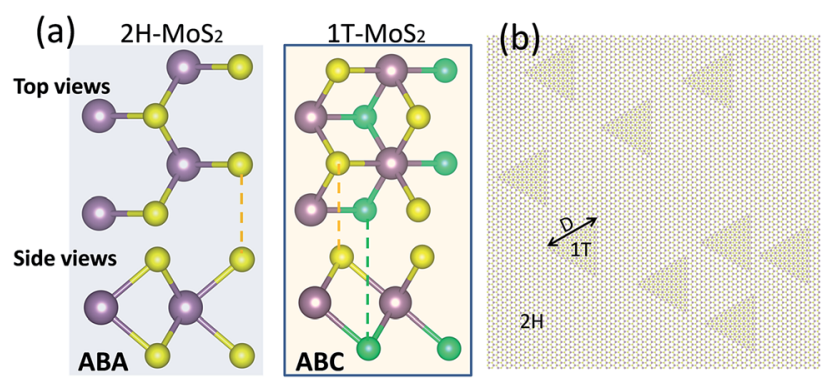

Fig. 1 (a) Top and side views of $2 \mathrm{H}$ and $1 \mathrm{~T}$ phases of single-layered $\mathrm{MoS}_{2}$. The $2 \mathrm{H}$ and $1 \mathrm{~T} M \mathrm{MS}_{2}$ show hexagonal lattices with ABA or ABC stacking sequences, respectively. (b) A periodic atomistic model of $2 \mathrm{H} /$ $1 \mathrm{~T}$ single-layer $\mathrm{MoS}_{2}$ heterostructure. The $1 \mathrm{~T}$ or $2 \mathrm{H}$ phases inside the heterostructures present triangular geometries ${ }^{18}$ with a characteristic domain size of " $D$ ". (a) " $\alpha$ "

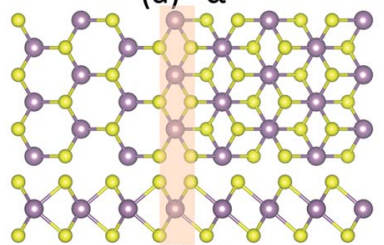

(c) " $\gamma$ "

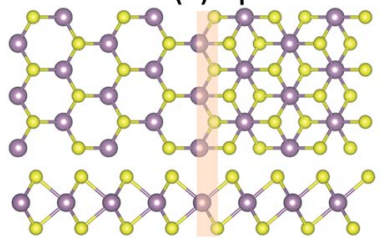

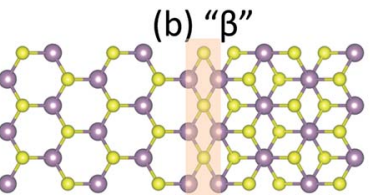

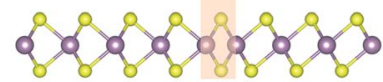

(d)
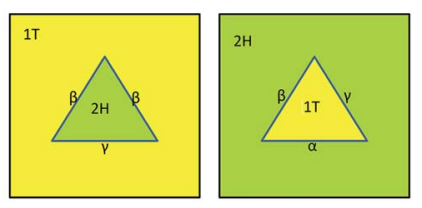

Fig. $2(\mathrm{a}-\mathrm{c})$ Top and side views of atomic structure of " $\alpha$ ", " $\beta$ " and " $\gamma$ " interfaces between $2 \mathrm{H}$ and $1 \mathrm{~T}$ phases. (d) Arrangement of different grain boundaries according to the configuration of single-layer $\mathrm{MoS}_{2}$ heterostructure, revealed by the experiment. ${ }^{18}$

experimentally synthesized samples change. ${ }^{18}$ In Fig. $2 d$, the arrangement of $\alpha, \beta$ and $\gamma$ interfaces for $2 \mathrm{H}$ inside $1 \mathrm{~T}$ and vice versa are illustrated.

In this investigation, we explored the thermal conductivity along all- $\mathrm{MoS}_{2}$ heterostructures by developing a combined atomistic-continuum multiscale method. ${ }^{26}$ In this approach, classical molecular dynamics simulations were carried out to compute thermal conductivities of the pristine $2 \mathrm{H}$ and $1 \mathrm{~T}$ phases and also to acquire the contact conductance along the $\alpha$, $\beta$ and $\gamma$ interfaces. Finally, the properties obtained by the atomistic simulations were used to construct macroscopic samples of $\mathrm{MoS}_{2}$ heterostructures using the finite element method. The details of molecular dynamics and finite element modeling are presented in detail as follows.

Molecular dynamics calculations in this study were performed using LAMMPS (Large-scale Atomic/Molecular Massively Parallel Simulator ${ }^{27}$ package. We used reactive empirical bond order (REBO) potential ${ }^{28,29}$ for the modeling of atomic interactions in the $\mathrm{MoS}_{2}$ structures. Worthy to note that we found that only ReaxFF ${ }^{25}$ and $\mathrm{REBO}^{28,29}$ forcefields can keep the constructed $\mathrm{MoS}_{2}$ structures stable. The non-equilibrium molecular dynamics (NEMD) method was employed to study the thermal conduction properties. Time increment of the NEMD simulation for the evaluation of thermal conductivity of the pristine films and thermal conductance of the interface boundaries was set to 0.2 fs and $0.1 \mathrm{fs}$, respectively. The NEMD modeling performed in this study is very similar to those we used in our recent works. ${ }^{30}$ In this approach, simulations were performed for samples with different lengths. For each sample, we applied periodic boundary condition along the planar directions to remove the effect of free atoms on the boundaries. All structures were first relaxed and equilibrated at the room temperature $(300 \mathrm{~K})$ using the Nosé-Hoover barostat and thermostat (NPT) method for 20 ps. For the NPT method, the damping parameters for the temperature and pressure were set at $10 \mathrm{fs}$ and $100 \mathrm{fs}$, respectively. Next, several rows of atoms at the two ends were fixed and the structures were further equilibrated using the Nosé-Hoover thermostat method (NVT). The 
simulation box was divided into several slabs and then as for the loading condition, we applied a temperature difference along the first and last slabs. ${ }^{30}$ In this case, the temperatures for these two slabs were controlled at the adjusted values using the NVT method, while the rest of the structure were simulated using the constant energy (NVE) method. We remind that the damping parameter for the all NVT steps in this work was set at 10 fs. To keep the imposed temperature difference during the simulation time, it is necessary to apply a constant heat-flux along the system in order to observe the steady-state heat transfer condition. As a result of the imposed temperature difference, a heat-flux is applied on the system by continuously adding energy to the atoms in the hot slab (with higher temperature) and removing energy from the atoms in the cold slab (with lower temperature). The applied heat-flux, $q_{\mathrm{x}}$, can be computed using the following relation:

$$
q_{\mathrm{x}}=\frac{1}{A} \frac{\mathrm{d} E}{\mathrm{~d} t}
$$

here $A$, is the cross sectional area of the $\mathrm{MoS}_{2}$ membranes assuming a thickness of $6.1 \AA$ (ref. 25) and $E$ is the energy values added or removed. Because of the applied temperature difference and imposed heat-flux, the steady-state heat transfer condition is finally achieved and a temperature gradient is established along the specimen length. The temperature at each slab was calculated using the following relation:

$$
T_{i(\text { slab })}=\frac{2}{3 N_{i} k_{\mathrm{B}}} \sum_{j} \frac{p_{j}^{2}}{2 m_{j}}
$$

where $T_{i(\text { (slab) }}$ is the temperature of $i^{\text {th }}$ slab, $N_{i}$ is the number of atoms in $i^{\text {th }}$ slab, $k_{\mathrm{B}}$ is the Boltzmann's constant, $m_{j}$ and $p_{j}$ are atomic mass and momentum of atom $j$, respectively. To evaluate the thermal conductivity, we performed the NEMD simulations for several nanoseconds and the temperature values at every slab were averaged. As it is shown in the Fig. 3a, by neglecting the initial jumps at the two end of the temperature profile, a linear relation is established $\left(\frac{\mathrm{d} T}{\mathrm{~d} x}\right.$ is constant). For the same sample, the net energy values added or removed from the structure are plotted in Fig. 3b. In this work, the energy values were recorded every 2 fs of simulation time and were averaged for every $40 \mathrm{fs}$ intervals to calculate the applied heat-flux. As illustrated in Fig. 3b, the amount of energy added to the hot reservoir and that removed from the cold reservoir are equal which implies that the total energy of the system is conserved. In addition, slopes of energy curves are linear meaning that the applied heat-flux is constant. According to the applied heat-flux and established temperature gradient, the thermal conductivity of pristine films, $k$, can be calculated using the one-dimensional form of the Fourier law:

$$
k=q_{\mathrm{x}} \frac{\mathrm{d} x}{\mathrm{~d} T}
$$

For the evaluation of thermal contact conductance, the established temperature difference, $\Delta T$ due to the presence of grain boundary was considered. In Fig. 3c, a sample of
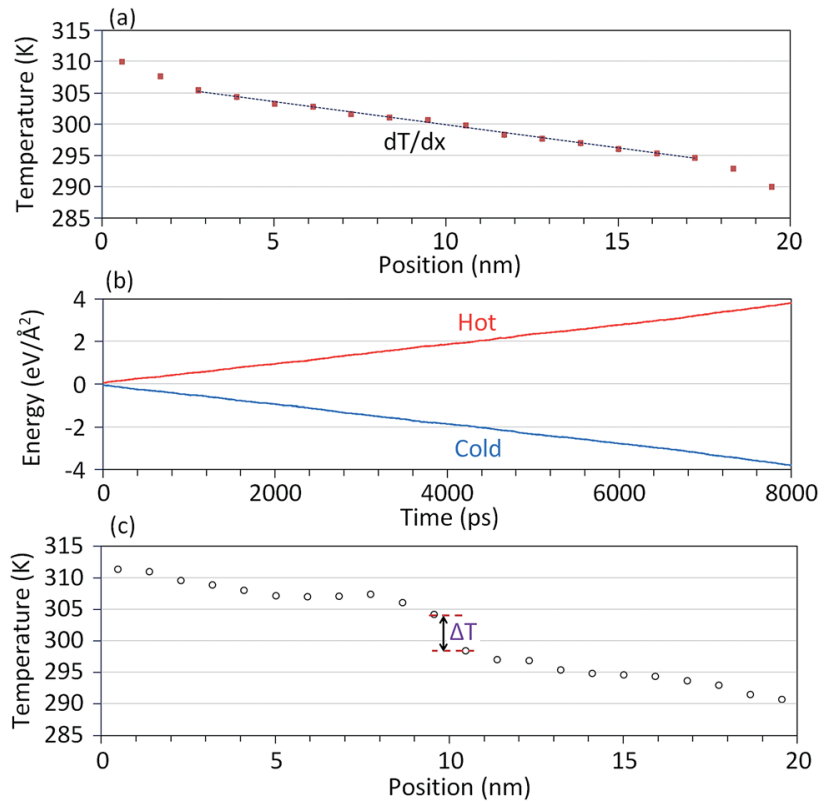

Fig. 3 (a and c) Established steady-state temperature profiles along the $20 \mathrm{~nm}$ samples as results of the applied temperature difference using the non-equilibrium molecular dynamics method. (a) For the pristine films, by neglecting the initial jumps a linear relation $(\mathrm{d} T / \mathrm{d} x)$ exist along the sample. (c) For the modeling of grain boundaries thermal contact conductance, a temperature jump $(\Delta T)$ occurs at the interface of $1 \mathrm{~T}$ and $2 \mathrm{H}$ phases (here we plotted the temperature profile for the " $\alpha$ " grain boundary). (b) Energy values added to the hot reservoir and removed from the cold reservoir during the NEMD simulation for the evaluation of the thermal conductivity of a pristine $1 \mathrm{~T} \mathrm{MoS}$ with length of $20 \mathrm{~nm}$.

temperature profile along a $1 \mathrm{~T} / 2 \mathrm{H}$ interface is depicted. As it can be observed, the temperature profile present a clear temperature difference in the middle of the sample where the $1 \mathrm{~T} / 2 \mathrm{H}$ interface is positioned. The interface conductance, $C_{\mathrm{i}}$ can be computed on the basis of the applied heat-flux, $q_{\mathrm{x}}$ and the established temperature difference, $\Delta T$ using the following relation:

$$
C_{\mathrm{i}}=\frac{q_{\mathrm{x}}}{\Delta T}
$$

Finally, the effective thermal conductivity of heterostructures was obtained using the finite element (FE) method. Finite element modeling in this study were carried out in ABAQUS/ standard by using python scripting. For the construction of continuum models of $\mathrm{MoS}_{2}$ heterostructure, according to the volume fraction and domain size of the secondary phase, we randomly distributed 1000 triangles in a square plane with a defined dimension of $L$. We used the NEMD results for the thermal conductivity of pristine films to introduce the thermal conductivities of each corresponding phase. Moreover, 1T/2H interface conductance values obtained by the NEMD method were used to introduce $1 \mathrm{~T} / 2 \mathrm{H}$ contact conductance properties in the same orders as those realized experimentally (Fig. 2d). ${ }^{18}$ In the modeling of thermal contact conductance elements in ABAQUS, one has two separate every two contacting faces. In 
this work, the master surface was chosen to be the one that is on the original phase and then the other contacting surface on the secondary phase was selected to be the slave surface. We used the free method for the meshing of the constructed heterostructures in ABAQUS using the combination of 3-node (DC2D3) and 4-node (DC2D4) linear heat transfer element types. For the loading condition, positive (inward) and negative (outward) heat-fluxes $\left(h_{\mathrm{f}}\right)$ were applied on the two opposite surfaces of the constructed models. The temperature of the outer surface of the cold surface (the surface in which the outward heat-flux is applied) was set to zero as the boundary condition. As a result of applied constant heat-flux, a steady-state temperature profile forms along the sample. The temperature difference along the specimen, $\Delta T$ was then computed which is proportional to the structure effective thermal conductivity, $k_{\text {eff }}$, through:

$$
k_{\mathrm{eff}}=h_{\mathrm{f}} \frac{L}{\Delta T}
$$

\section{Results and discussions}

We performed NEMD simulations for the pristine $2 \mathrm{H}$ and $1 \mathrm{~T}$ phases and $1 \mathrm{~T} / 2 \mathrm{H}$ interfaces for samples with different lengths to explore the length effect on the predicted thermal conduction properties. For the pristine $2 \mathrm{H}$ and $1 \mathrm{~T} \mathrm{MoS}_{2}$, we found increasing trends in the thermal conductivity by increasing the length $(L)$ of the structure. As a common approach, the thermal conductivity of membranes with infinite length can be obtained by extrapolation of results for the samples with finite lengths. A simple approach is to define an effective phonon mean free path $\left(\Lambda_{\text {eff }}\right)$ as $1 / \Lambda_{\text {eff }}=1 / \Lambda+1 / L$. Since the thermal conductivity is proportional to $\Lambda_{\text {eff }}$, the thermal conductivity of the infinite system can be obtained by extrapolating to $1 / L=0 .{ }^{31}$ In Fig. 4 , the NEMD results for the inverse of thermal conductivity of defect-free $1 \mathrm{~T}$ and $2 \mathrm{H} \mathrm{MoS}$ as a function of length inverse are illustrated. According to the explained methodology, we fitted lines to the NEMD results shown in Fig. 4, in order to obtain the thermal conductivity of the sheets with infinite length. This way, the thermal conductivity of pristine $2 \mathrm{H}$ and $1 \mathrm{~T} \mathrm{MoS}_{2}$ at room temperature are calculated to be $40 \pm 4 \mathrm{~W} \mathrm{~m}^{-1} \mathrm{~K}^{-1}$ and

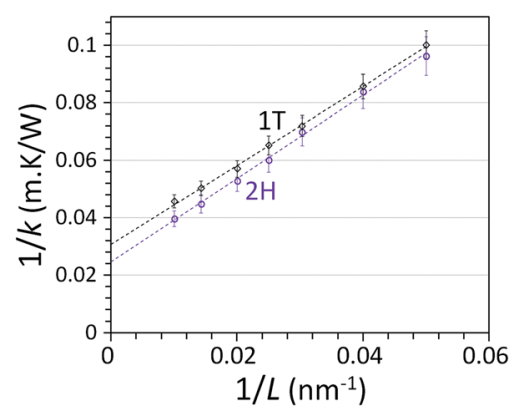

Fig. 4 Calculated inverse of thermal conductivity of defect-free 1T and $2 \mathrm{H} \mathrm{MoS}_{2}$ as a function of length inverse. By fitting linear relations (as shown here with dashed lines), thermal conductivity of the pristine films with infinite lengths were obtained.
$32 \pm 3 \mathrm{~W} \mathrm{~m}^{-1} \mathrm{~K}^{-1}$, respectively. There have been numerous experimental reports for lattice thermal conductivity of semiconducting $\mathrm{MoS}_{2} \cdot{ }^{32,33}$ In these preliminary experimental works, thermal conductivity of few-layer $2 \mathrm{H} \mathrm{MoS}_{2}$ were measured to be $34.5 \pm 4 \mathrm{~W} \mathrm{~m}^{-1} \mathrm{~K}^{-1}$ (ref. 32) and $\sim 52 \mathrm{~W} \mathrm{~m}^{-1} \mathrm{~K}^{-1} \cdot{ }^{33}$ Moreover, recently the thermal conductivity of high-quality bulk $2 \mathrm{H} \mathrm{MoS}$ were experimentally measured to be in a range of $85-110 \mathrm{~W} \mathrm{~m}^{-1}$ $\mathrm{K}^{-1}{ }^{34}$ Considering the wide range of experimental results for the thermal conductivity of $2 \mathrm{H} \mathrm{MoS}_{2}$, our NEMD results based on the REBO potential yields a thermal conductivity with acceptable accuracy. It should be emphasized that our aim is to study the relative thermal conductivity of all- $\mathrm{MoS}_{2}$ heterostructures and not to exactly report the thermal conductivity. We note that to the best of our knowledge the thermal conductivity of metallic $1 \mathrm{~T} \mathrm{MoS}_{2}$ has been studied neither experimentally nor theoretically. Based on our NEMD results, the thermal conductivity of $1 \mathrm{~T} \mathrm{MoS}_{2}$ is only around $20 \%$ smaller than that for the $2 \mathrm{H} \mathrm{MoS}_{2}$. We also analyzed the length effect on the thermal contact conductance of the $1 \mathrm{~T} / 2 \mathrm{H}$ grain boundaries and in this case we realized that the length effect is negligible. This can be explained due to the fact that the phonon-defects scattering along the $1 \mathrm{~T} / 2 \mathrm{H}$ interface as the main factor that determines the thermal resistance, phonon-phonon scattering plays a marginal role here. Based on our classical molecular dynamics modeling, thermal conductance of $\alpha, \beta$ and $\gamma$ grain boundaries were obtained to be $0.75 \pm 0.1 \mathrm{GW} \mathrm{m}^{-2} \mathrm{~K}^{-1}, 1.1 \pm$ $0.15 \mathrm{GW} \mathrm{m}^{-2} \mathrm{~K}^{-1}$ and $0.66 \pm 0.1 \mathrm{GW} \mathrm{m}^{-2} \mathrm{~K}^{-1}$ respectively.

In order to understand the underlying mechanism resulting in different thermal conduction properties along $\mathrm{MoS}_{2}$ structures, we calculated the phonon density of states (PDOS). In this case, we simulate structures at room temperature under the NVE method for $120 \mathrm{ps}$ in which atomic velocities were recorded every 1 fs. By post-processing the trajectories the PDOS were calculated. The PDOS was computed by calculating the Fourier transform of the velocity autocorrelation function, as follows:

$$
\operatorname{PDOS}(\omega)=\int_{0}^{\infty} \frac{\langle v(t) v(0)\rangle}{\langle v(0) v(0)\rangle} \mathrm{e}^{-\mathrm{i} \omega t} \mathrm{~d} t
$$

where $\omega$ is the frequency and the $v$ is the atomic velocity. In Fig. 5 , we illustrate the computed PDOS for the Mo and S atoms separately and here the total PDOS was obtained by summing those for the native atoms. For the pristine $2 \mathrm{H}$ and $1 \mathrm{~T} \mathrm{MoS}_{2}$ phases, one can observe very similar trends. However, in the $1 \mathrm{~T}$ phase the characteristic peaks are slightly damped and shifted to the left with respect to those in pristine $2 \mathrm{H} \mathrm{MoS}$. This suggest small phonon softening which could decrease the phonon group velocity and explain the lower thermal conductivity. We also remind that based on our recent molecular dynamics study the elastic modulus of $2 \mathrm{H} \mathrm{MoS}$ is about two times higher than that in $1 \mathrm{~T} \mathrm{MoS}_{2} \cdot{ }^{25}$ Along the grain boundaries, as a result of phonondefect scatterings, the main peaks in the PDOS are considerably damped and broadened. In comparison with the pristine films, in these cases more phonons are excited at lower frequencies than $6 \mathrm{THz}$. The enhanced vibrational states at low frequencies suggest higher phonon scattering rates and thus lower interface conductance. This way, the excitation of low frequency phonons are most and least along $\beta$ and $\gamma$ grain boundaries, respectively. 
This can explain our NEMD results which revealed the highest and lowest thermal conductance along the $\beta$ and $\gamma$ grain boundaries, respectively.

The thermal conductivity of heterostructures strongly correlates to the volume concentration of the additive phase. In this work we studied samples with $5 \%$ and $20 \%$ concentrations of the secondary phase. We considered only two concentrations of $20 \%$ and $5 \%$ because they can represent samples with high and medium concentrations, respectively. The main factor that may dominate the heat conduction along the $\mathrm{MoS}_{2}$ heterostructures is the domain size of the secondary phase and such that we considered a wide range from $1 \mathrm{~nm}$ to $1000 \mathrm{~nm}$ to elaborately discuss this effect. In Fig. 6, the effective thermal conductivity of various $\mathrm{MoS}_{2}$ heterostructures as a function of domain size predicted by the NEMD/FE multiscale approach are illustrated. We reported the normalized thermal conductivity of heterostructures with respect to the thermal conductivity of

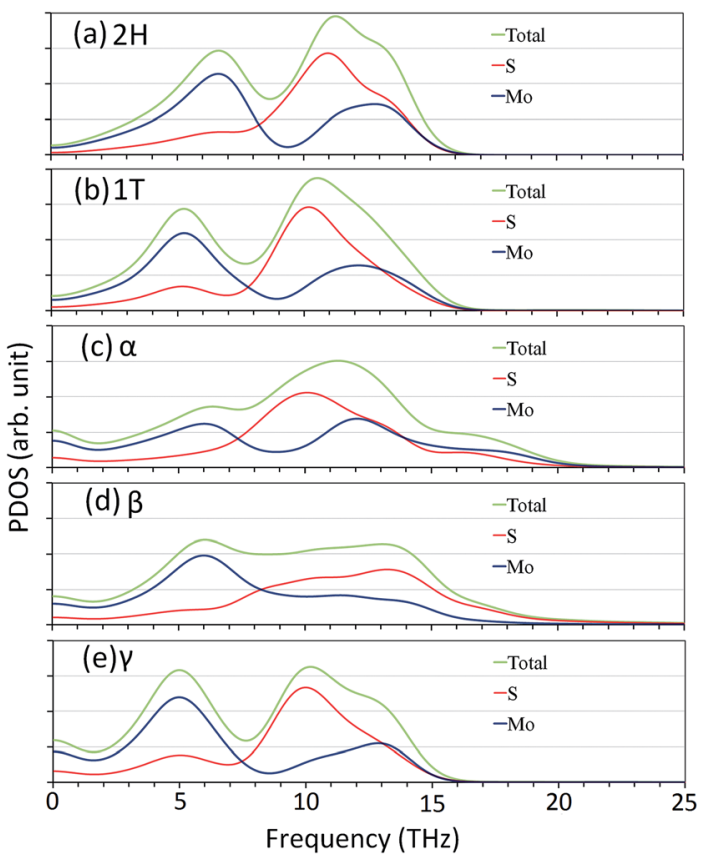

Fig. 5 Calculated vibrational phonon density of states (PDOS) for pristine $1 \mathrm{~T}$ and $2 \mathrm{H} \mathrm{MoS}_{2}$ and $\alpha, \beta$ and $\gamma 1 \mathrm{~T} / 2 \mathrm{H}$ interfaces.

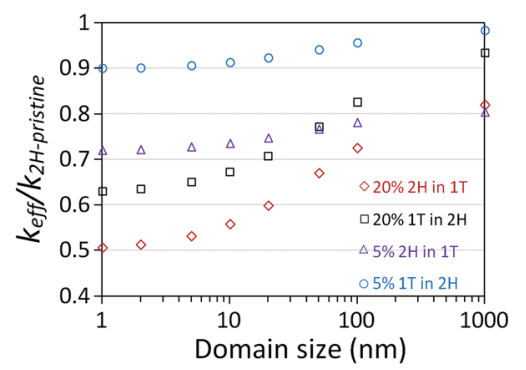

Fig. 6 Multiscale results for the effective thermal conductivity of different $\mathrm{MoS}_{2}$ heterostructures as a function of domain size. The results are normalized with respect to the thermal conductivity of pristine $2 \mathrm{H} \mathrm{MOS}_{2}\left(k_{2 \mathrm{H} \text {-pristine }}\right)$. pristine $2 \mathrm{H} \mathrm{MoS}_{2}$ in order to comparatively investigate the heat conduction. As illustrated in Fig. 6, the effective thermal conductivity of $\mathrm{MoS}_{2}$ heterostructures keep increasing by increasing the domain size. This enhancement in the thermal conductivity by increasing the domain size was found to be considerable for the heterostructures with the domain sizes smaller than $100 \mathrm{~nm}$. This finding reveals the considerable importance of the $1 \mathrm{~T} / 2 \mathrm{H}$ interfaces thermal conductance when the domain sizes are at nanoscale. Since the thermal conductivity of $2 \mathrm{H}$ phase is higher than that for $1 \mathrm{~T}$ $\mathrm{MoS}_{2}$, one may expect higher effective thermal conductivity for the heterostructures with higher concentration of $2 \mathrm{H}$ phase inside the 1T. According to our results, this expectation is however valid only for the heterostructures with domain sizes around or higher than 1 $\mu \mathrm{m}$. In this case, despite of the higher concentration of the phase with superior thermal conductivity ( $2 \mathrm{H}$ phase), the increase in the thermal resistance due to the increase of the $1 \mathrm{~T} / 2 \mathrm{H}$ interfaces leads to a decline in the effective thermal conductivity, particularly for the heterostructures with domain sizes smaller than $100 \mathrm{~nm}$. This way, based on our multiscale modeling the effect of $1 \mathrm{~T} / 2 \mathrm{H}$ grain boundary conductance on the thermal conductivity of $\mathrm{MoS}_{2}$ heterostructures become negligible only when the domain sizes are around micrometers. Our multiscale results reveal that the thermal conductivity of $\mathrm{MoS}_{2}$ can be finely tuned by fabrication of heterostructures. Moreover, we predict that in comparison with the pristine $2 \mathrm{H}$ phase the thermal conductivity of $\mathrm{MoS}_{2}$ heterostructure cannot be substantially suppressed. This finding is important and reveals that the thermal management of nanodevices made from $\mathrm{MoS}_{2}$ heterostructure may not be considerably declined in comparison with those made only from pristine $2 \mathrm{H} \mathrm{MoS}_{2}$.

In Fig. 7, samples of modeling results for the calculated steady-state temperature and heat-flux profiles for $\mathrm{MoS}_{2}$ heterostructures constructed with $20 \% 1 \mathrm{~T}$ phase inside $2 \mathrm{H} \mathrm{MoS}_{2}$ with domain sizes of $1 \mathrm{~nm}$ and $100 \mathrm{~nm}$ are compared. For the sample with domain size of $1 \mathrm{~nm}$, the temperature distribution inside $1 \mathrm{~T}$ phase are found to be primarily constant (Fig. 7d inset) and the temperature changes are mainly occurring at the $1 \mathrm{~T} / 2 \mathrm{H}$ interfaces. In this case, the secondary $1 \mathrm{~T}$ triangular domains are almost not participating in the heat-flux transfer

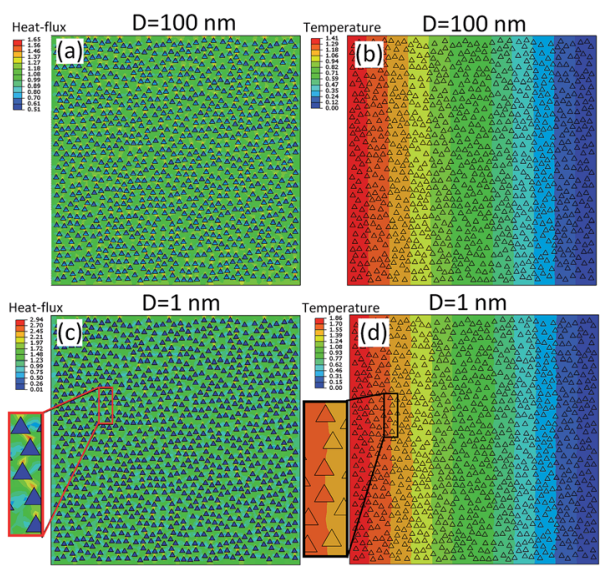

Fig. 7 Comparison of the effect of domain size effect on the temperature and heat-flux distributions for $\mathrm{MoS}_{2}$ heterostructures constructed with $20 \% 1 \mathrm{~T}$ phase inside $2 \mathrm{H} \mathrm{MoS}_{2}$. 
(Fig. 7c). Since the $1 \mathrm{~T} / 2 \mathrm{H}$ interface resistance effect dominates the thermal conductivity for small domain sizes, the heat-flux is transferred only though the original $2 \mathrm{H}$ phase and the $1 \mathrm{~T}$ phase are almost acting as voids. On the other hand, for the heterostructures with large domain sizes, the temperature changes are more uniformly happening throughout the structure (Fig. 7b) which implies that the effect of $1 \mathrm{~T} / 2 \mathrm{H}$ interface resistance is substantially weakened. In this case, although the $2 \mathrm{H}$ phase is more engaged in the heat-flux transfer, the $1 \mathrm{~T}$ triangles also actively participate in the heat conduction.

\section{Conclusion}

We proposed a combined atomistic-continuum multiscale modeling to explore the effective thermal conductivity of all$\mathrm{MoS}_{2}$ single-layer heterostructures. In this approach, nonequilibrium molecular dynamics (NEMD) method was employed to compute the thermal conductivity of pristine semiconducting $(2 \mathrm{H})$ and metallic (1T) $\mathrm{MoS}_{2}$ phases and also to calculate the thermal contact conductance between $1 \mathrm{~T}$ and $2 \mathrm{H}$ phases. In order to investigate the effective thermal conductivity of heterostructures at larger scales, we constructed relatively large continuum models of $\mathrm{MoS}_{2}$ heterostructures using the finite element (FE) approach. In this regard, acquired properties by the molecular dynamics simulations were used to introduce thermal conductivity of the representative phases as well as the corresponding interfaces thermal conductance. Our classical modeling results based on the REBO potential for the pristine $2 \mathrm{H} \mathrm{MoS}$ yields a thermal conductivity of around $40 \pm 4 \mathrm{~W} \mathrm{~m}^{-1}$ $\mathrm{K}^{-1}$, with acceptable accuracy as compared with experimental results. Moreover, the thermal conductivity of metallic $\mathrm{MoS}_{2}$ was predicted to be only around $20 \%$ smaller than that for the pristine semiconducting $\mathrm{MoS}_{2}$. The thermal conductance of $\alpha$, $\beta$ and $\gamma$ interfaces between $1 \mathrm{~T}$ and $2 \mathrm{H}$ phases were estimated to be $0.75 \pm 0.1 \mathrm{GW} \mathrm{m}^{-2} \mathrm{~K}^{-1}, 1.1 \pm 0.15 \mathrm{GW} \mathrm{m}^{-2} \mathrm{~K}^{-1}$ and $0.66 \pm$ $0.1 \mathrm{GW} \mathrm{m} \mathrm{m}^{-2} \mathrm{~K}^{-1}$, respectively. NEMD/FE multiscale results reveal the significance of $1 \mathrm{~T} / 2 \mathrm{H}$ interfaces thermal conductance effect when the domain sizes are at nanoscale. It was shown that the effective thermal conductivity of heterostructures increases by increasing the domain size. We explain that the thermal conductivity of $\mathrm{MoS}_{2}$ can be finely tuned by fabrication of heterostructures. More importantly, we predicted that owing to the strong thermal coupling of $1 \mathrm{~T} / 2 \mathrm{H}$ interfaces the effective thermal conductivity of $\mathrm{MoS}_{2}$ heterostructure remains close to that of pristine $2 \mathrm{H}$ phase. This finding reveals that the thermal management of nanodevices made from $\mathrm{MoS}_{2}$ heterostructure would not be declined in comparison with those made from pristine $2 \mathrm{H} \mathrm{MoS}$. In response to the remarkably fast growth and interests in the field of $2 \mathrm{D}$ materials, the proposed multiscale approach can be considered to efficiently explore the effective thermal conductivity of various $2 \mathrm{D}$ heterostructures.

\section{Acknowledgements}

BM and TR greatly acknowledge the financial support by European Research Council for COMBAT project (Grant number 615132).

\section{References}

1 K. S. Novoselov, A. K. Geim, S. V Morozov, D. Jiang, Y. Zhang, S. V. Dubonos, et al., Electric field effect in atomically thin carbon films., Science, 2004, 306, 666-669, DOI: 10.1126/ science.1102896.

2 A. K. Geim and K. S. Novoselov, The rise of graphene, Nat. Mater., 2007, 6, 183-191, DOI: 10.1038/nmat1849.

3 F. Schedin, A. K. Geim, S. V. Morozov, E. W. Hill, P. Blake, M. I. Katsnelson, et al., Detection of individual gas molecules adsorbed on graphene., Nat. Mater., 2007, 6, 652-655, DOI: 10.1038/nmat1967.

4 A. H. Castro Neto, N. M. R. Peres, K. S. Novoselov, A. K. Geim and F. Guinea, The electronic properties of graphene, Rev. Mod. Phys., 2009, 81, 109-162, DOI: 10.1103/RevModPhys.81.109.

5 S. Ghosh, W. Bao, D. L. Nika, S. Subrina, E. P. Pokatilov, C. N. Lau, et al., Dimensional crossover of thermal transport in few-layer graphene., Nat. Mater., 2010, 9, 555558, DOI: $10.1038 /$ nmat2753.

6 C. Lee, X. Wei, J. W. Kysar and J. Hone, Measurement of the Elastic Properties and Intrinsic Strength of Monolayer Graphene, Science, 2008, 321, 385-388, DOI: 10.1126/ science.1157996.

7 B. Mortazavi, Z. Fan, L. F. C. Pereira, A. Harju and T. Rabczuk, Amorphized graphene: A stiff material with low thermal conductivity, Carbon, 2016, 103, 318-326, DOI: 10.1016/j.carbon.2016.03.007.

8 G. R. Berdiyorov and M. E. A. Madjet, First-principles study of electronic transport and optical properties of pentagraphene, penta-SiC ${ }_{2}$ and penta- $\mathrm{CN}_{2}, R S C A d v ., 2016,6$, 50867-50873, DOI: 10.1039/c6ra10376f.

9 B. Mortazavi, G. Cuniberti and T. Rabczuk, Mechanical properties and thermal conductivity of graphitic carbon nitride: A molecular dynamics study, Comput. Mater. Sci., 2015, 99, 285-289, DOI: 10.1016/j.commatsci.2014.12.036.

10 A. A. Khatibi and B. Mortazavi, A study on the nanoindentation behaviour of single crystal silicon using hybrid MD-FE method, Adv. Mater. Res., 2008, 32, 259-262.

11 K. Watanabe, T. Taniguchi and H. Kanda, Direct-bandgap properties and evidence for ultraviolet lasing of hexagonal boron nitride single crystal, Nat. Mater., 2004, 3, 404-409, DOI: $10.1038 /$ nmat1134.

12 Y. Kubota, K. Watanabe, O. Tsuda and T. Taniguchi, Deep ultraviolet light-emitting hexagonal boron nitride synthesized at atmospheric pressure, Science, 2007, 317, 932-934, DOI: 10.1126/science.1144216.

13 L. Song, L. Ci, H. Lu, P. B. Sorokin, C. Jin, J. Ni, et al., Large scale growth and characterization of atomic hexagonal boron nitride layers, Nano Lett., 2010, 10, 3209-3215, DOI: 10.1021/nl1022139.

14 L. Liu, J. Park, D. a. Siegel, K. F. McCarty, K. W. Clark, W. Deng, et al., Heteroepitaxial Growth of Two-Dimensional Hexagonal Boron Nitride Templated by Graphene Edges, Science, 2014, 343, 163-167, DOI: 10.1126/science.1246137.

15 Z. Liu, L. Ma, G. Shi, W. Zhou, Y. Gong, S. Lei, et al., In-plane heterostructures of graphene and hexagonal boron nitride 
with controlled domain sizes, Nat. Nanotechnol., 2013, 8, 119-124, DOI: 10.1038/nnano.2012.256.

16 B. Radisavljevic, A. Radenovic, J. Brivio, V. Giacometti and A. Kis, Single-layer $\mathrm{MoS}_{2}$ transistors, Nat. Nanotechnol., 2011, 6, 147-150, DOI: 10.1038/nnano.2010.279.

17 Q. H. Wang, K. Kalantar-Zadeh, A. Kis, J. N. Coleman and M. S. Strano, Electronics and optoelectronics of twodimensional transition metal dichalcogenides, Nat. Nanotechnol., 2012, 7, 699-712, DOI: 10.1038/nnano.2012.193.

18 Y.-C. Lin, D. O. Dumcenco, Y.-S. Huang and K. Suenaga, Atomic mechanism of the semiconducting-to-metallic phase transition in single-layered $\mathrm{MoS}_{2}$, Nat. Nanotechnol., 2014, 9, 391-396, DOI: 10.1038/nnano.2014.64.

19 L. F. Mattheiss, Band structures of transition-metaldichalcogenide layer compounds, Phys. Rev. B: Condens. Matter Mater. Phys., 1973, 8, 3719-3740, DOI: 10.1103/ PhysRevB.8.3719.

20 F. Wypych and R. Schöllhorn, 1T- $\mathrm{MoS}_{2}$, a new metallic modification of molybdenum disulfide, J. Chem. Soc., Chem. Commun., 1992, 1386-1388.

21 Y. Gao, Q. Liu and B. Xu, Lattice Mismatch Dominant Yet Mechanically Tunable Thermal Conductivity in Bilayer Heterostructures, ACS Nano, 2016, 10, 5431-5439, DOI: 10.1021/acsnano.6b01674.

22 M.-H. Chiu, C. Zhang, H.-W. Shiu, C.-P. Chuu, C.-H. Chen, C. Y. S. Chang, et al., Determination of band alignment in the single-layer $\mathrm{MoS}_{2} / \mathrm{WSe}_{2}$ heterojunction, Nat. Commun., 2015, 6, 7666, DOI: 10.1038/ncomms8666.

23 R. Ganatra and Q. Zhang, Few-layer $\mathrm{MoS}_{2}$ : A promising layered semiconductor, ACS Nano, 2014, 8, 4074-4099, DOI: $10.1021 / \mathrm{nn} 405938 \mathrm{z}$.

24 N. B. Le, T. D. Huan and L. M. Woods, Interlayer Interactions in van der Waals Heterostructures: Electron and Phonon Properties, ACS Appl. Mater. Interfaces, 2016, 8, 6286-6292, DOI: 10.1021/acsami.6b00285.

25 B. Mortazavi, A. Ostadhossein, T. Rabczuk and A. C. T. van Duin, Mechanical response of all-MoS $\mathrm{Mos}_{2}$ single-layer heterostructures: a ReaxFF investigation, Phys. Chem. Chem. Phys., 2016, 18, 23695-23701, DOI: 10.1039/c6cp03612k.
26 B. Mortazavi, J. Bardon, J. A. S. Bomfim and S. Ahzi, A statistical approach for the evaluation of mechanical properties of silica/epoxy nanocomposite: Verification by experiments, Comput. Mater. Sci., 2012, 59, 108-113, DOI: 10.1016/j.commatsci.2012.03.002.

27 S. Plimpton, Fast Parallel Algorithms for Short-Range Molecular Dynamics, J. Comput. Phys., 1995, 117, 1-19, DOI: $10.1006 /$ jcph.1995.1039.

28 T. Liang, S. R. Phillpot and S. B. Sinnott, Parametrization of a reactive many-body potential for Mo-S systems, Phys. Rev. B: Condens. Matter Mater. Phys., 2009, 79, 245110.

29 K. Q. Dang, J. P. Simpson and D. E. Spearot, Phase transformation in monolayer molybdenum disulphide $\left(\mathrm{MoS}_{2}\right)$ under tension predicted by molecular dynamics simulations, Scr. Mater., 2014, 76, 41-44, DOI: 10.1016/ j.scriptamat.2013.12.011.

30 B. Mortazavi, O. Rahaman, T. Rabczuk and L. F. C. Pereira, Thermal conductivity and mechanical properties of nitrogenated holey graphene, Carbon, 2016, 106, 1-8, DOI: 10.1016/j.carbon.2016.05.009.

31 P. K. Schelling, S. R. Phillpot and P. Keblinski, Comparison of atomic-level simulation methods for computing thermal conductivity, Phys. Rev. B: Condens. Matter Mater. Phys., 2002, 65, 1-12, DOI: 10.1103/PhysRevB.65.144306.

32 R. Yan, J. R. Simpson, S. Bertolazzi, J. Brivio, M. Watson, $\mathrm{X}$. Wu, et al., Thermal conductivity of monolayer molybdenum disulfide obtained from temperaturedependent Raman spectroscopy, ACS Nano, 2014, 8, 986993, DOI: 10.1021/nn405826k.

33 S. Sahoo, A. P. S. Gaur, M. Ahmadi, M. J. F. Guinel and R. S. Katiyar, Temperature-dependent Raman studies and thermal conductivity of few-layer $\mathrm{MoS}_{2}, J$. Phys. Chem. C, 2013, 117, 9042-9047, DOI: 10.1021/jp402509w.

34 J. Liu, G. M. Choi and D. G. Cahill, Measurement of the anisotropic thermal conductivity of molybdenum disulfide by the time-resolved magneto-optic Kerr effect, J. Appl. Phys., 2014, 116, 4904513. 